\title{
Photoconductivity mechanism in structures with Ge-nanoclusters grown on $\mathrm{Si}(\mathbf{1 0 0})$ surface
}

\author{
Ye.Ye. Melnichuk ${ }^{1}$, Yu.V. Hyrka ${ }^{1}$, S.V. Kondratenko ${ }^{1}$, Yu.N. Kozyrev ${ }^{2}$, V.S. Lysenko ${ }^{3}$ \\ ${ }^{I}$ Taras Shevchenko National University of Kyiv, 64/13, Volodymyrska str., 01601 Kyiv, Ukraine \\ ${ }^{2}$ O. Chuiko Institute of Surface Chemistry, 17, Generala Naumova str., 03164 Kyiv, Ukraine \\ ${ }^{3}$ V. Lashkaryov Institute of Semiconductor Physics, NAS of Ukraine, 03028 Kyiv, Ukraine
}

\begin{abstract}
Interband optical transitions in the epitaxial $\mathrm{Si} / \mathrm{Ge}$ heterostructures with $\mathrm{Ge}$ nanoislands grown on $\mathrm{Si}(100)$ surface were investigated using photocurrent spectroscopy. The mechanism of photoconductivity was discussed. It was shown that electron transitions from the ground state of the valence band in a quantum dot to the conduction band of $\mathrm{Si}$ surrounding make the main contribution into monopolar photoconductivity below the fundamental absorption edge of crystalline Si. Photoexcited holes were found to be localized in Ge nanoislands inducing the lateral conductivity changes in the near-surface depletion layer of $p$-Si substrate due to the field-effect.
\end{abstract}

Keywords: Ge-nanocluster, photoconductivity, surface potential, quantum dots.

Manuscript received 19.02.14; revised version received 29.05.14; accepted for publication 29.10.14; published online 10.11.14.

\section{Introduction}

Germanium nanoclusters grown on/in silicon have been successfully applied in new optoelectronic, and memory devices. Due to spatial confinement of charge carrier's motion in one, two or three directions, respectively, such nanostructures have unique fundamental properties and technological applications $[1,2]$. Of particular interest is attracted by nanoelectronic devices and systems grown using epitaxy methods - vapor-phase, molecular-beam and liquid-phase - in which the formation and spatial arrangement of nanoscale elements was carried out using the effects of self-organization.

In the heterosystem $\mathrm{Si} / \mathrm{Ge}$ with nanoislands distributed across the surface of inherent non-uniform field of mechanical stresses. Interfaces and their quantum-size classes, wetting layer (WL) heterogeneity leads to spatial heterogeneity of local electro-physical 
properties of $\mathrm{Ge}$ nanoclusters and induced spatial variation of the electrostatic potential. These features, expectedly, will have an impact on the transport of charge carriers along the epitaxial layers.

Heterojunctions $\mathrm{Si} / \mathrm{Ge}$ are referred to the second type, in which there is a limitation of motion of holes in Ge nanoclusters. That's why Ge nanoclusters can be considered as a long-term trap for holes, charge which a due to downward band bending in the underlying $\mathrm{Si}$.

Semiconductor heterostructures and especially semiconductor heterostructures with low-dimensional objects, including quantum wells, quantum wires and quantum dots, currently comprise the object of intensive study $[1,3]$. Knowledge of the electronic spectrum, transport, recombination, and photogeneration in selforganized nanostructures is essential for creation of novel electronic and photonic devices.

Low-dimensional $\mathrm{Ge} / \mathrm{Si}$ heterostructures have attracted considerable research interest in recent years, due to their significant potential to impact new electronic devices that are compatible with the available silicon technology. Optoelectronic devices based on SiGe dots grown on a $\mathrm{Si}$ substrate have been already proposed $[4,5]$. The low-dimensional silicon-germanium alloys have a wide range of applications, including quantum dot IR photodetectors, memory cells and spintronic devices. Widespread application of this system is arrangement of SiGe quantum dots in the space-charge region of heterojunctions, Schottky diodes, $p$ - $n$ junctions or metal-oxide-semiconductor structures.

\section{Experimental}

The molecular beam epitaxy (MBE) technique ("Katun'B" set-up, produced in Novosibirsk, Russia) was used to prepare multilayer Ge-Si(100) nanocluster arrays with islands of various sizes and surface density. The (100) oriented wafers of $n-\mathrm{Si}$ with 7.5 and $20 \mathrm{Ohm} \cdot \mathrm{cm}$ resistivity and diameter of $76 \mathrm{~mm}$ were used as substrates. In order to prepare multilayer quantum dot systems with regular nanoisland distribution over the substrate surface, we have proposed to use a system of $\mathrm{Si}_{1-x} \mathrm{Ge}_{x}$ intermediate layers with a sub-critical thickness [5]. The Ge mole fraction $x$ was gradually increased from layer-to-layer grown at gradually decreasing substrate temperature started from $T_{s}=500{ }^{\circ} \mathrm{C}$. The growth process, in particular the moment of the $2 D \rightarrow 3 D$ transition in the Stranski-Krastanov growth regime, was controlled via RHEED (reflection high energy electron diffraction). To study the surface morphology, atomic force microscopy (AFM) measurements were carried out using an Ntegra AFM from NT-MDT with a closed loop scanner. Standard Si cantilevers with tips having a half opening angle of $10^{\circ}$ were employed as probes. The growth of each Si intermediate layer was continued until a high-contrast $\mathrm{Si}(100) 2 \times 1$ RHEED pattern typical of clean Si was produced. Thus, the multilayer Ge-Si(100) nanocluster arrays were grown at the temperature $T_{s}=$ $500{ }^{\circ} \mathrm{C}$.

The Stranski-Krastanow growth of Ge nanoislands on $\mathrm{Si}(001)$ surface is an intermediary process characterized by both 2D WL and 3D island formation. Transition from the layer-by-layer epitaxy to nanoisland structure growth occurs at a critical layer thickness which is highly dependent on surface energies and lattice parameters. Germanium nanoclusters grown on/in silicon or silicon dioxide have been successfully applied in new nanoelectronic, optoelectronic and memory devices due to quantum confinement effect and possibility of integration within Si-based technology.

Micro-Raman scattering spectra of the investigated structures were recorded at room temperature using automated Raman diffraction spectrometer T-64000 Horiba Jobin-Yvon equipped with CCD detector. The line $488 \mathrm{~nm}$ of Ar-Kr laser of $3 \mathrm{~mW}$ was used for excitation. Raman spectra were measured for the geometry $z(x, y)-x$, where axes $x, y, z$ correspond to [100], [010] and [001] crystallographic directions, correspondingly. Ohmic $\mathrm{Au}-\mathrm{Si}$ contacts of rectangular shape and dimensions of $4 \times 1 \mathrm{~mm}$ were welded into epitaxial layers at $370{ }^{\circ} \mathrm{C}$ for lateral photoconductivity measurements. The distance between contacts on the sample surface was $5 \mathrm{~mm}$. Current-voltage characteristics of the structures studied were found to be linear in the range from $-10 \mathrm{~V}$ to $+10 \mathrm{~V}$ at temperatures between 50 and $290 \mathrm{~K}$. Lateral photoconductivity spectra were measured at excitation energies ranging from 0.48 up to $1.7 \mathrm{eV}$ under illumination with a $250-\mathrm{W}$ halogen lamp. The corresponding direct photocurrent signal was registered using a standard amplification technique. Spectral dependences were normalized to the constant number of exciting quanta using a non-selective pyroelectric detector.

\section{Results}

Fig. 1a shows AFM image of the top layer of a typical sample with one layer of nanoislands as large scatters significant in size. The figure shows that the surface contains nanoislands with the basic sizes about $98 \mathrm{~nm}$ and a height of about $15 \mathrm{~nm}$. The average surface density of nanoislands is $\sim 10^{10} \mathrm{~cm}^{-2}$. Composition and values of elastic strains in investigated $\mathrm{Ge} / \mathrm{Si}$ heterostructures were estimated using Raman spectroscopy. Typical Raman spectrum of $\mathrm{Ge} / \mathrm{Si}$ heterostructure containing 5 layers of Ge quantum dots is given in Fig. 1b. It contains phonon bands corresponding to $\mathrm{Ge}-\mathrm{Ge}, \mathrm{Si}-\mathrm{Ge}$ and $\mathrm{Si}-\mathrm{Si}$ vibrations, which is typical for $\mathrm{SiGe}$ heterostructures with nanoislands, which makes it possible to estimate content and strain values for Ge nanoislands [9]. Thus, Ge mole fraction and elastic strains in Ge nanoislands were found to be $x=0.91 \pm 0.02$ and, $\varepsilon_{x x}=-0.01$, correspondingly. 

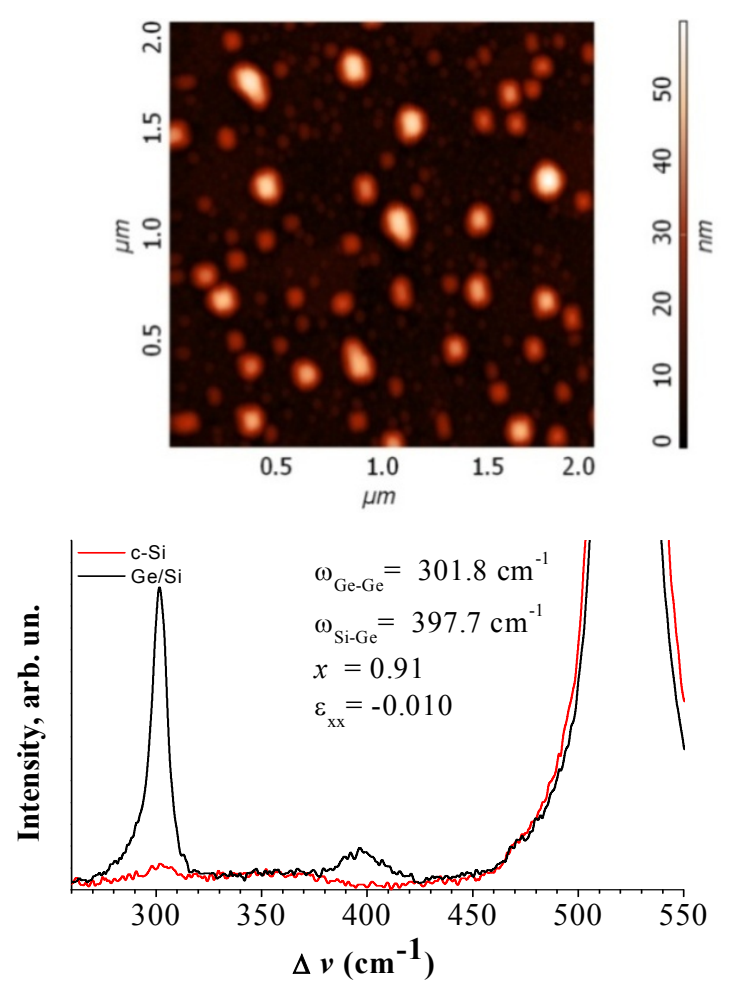

a)

b)

Fig. 1. The AFM image of the surface of Ge nanoislands grown using $\mathrm{MBE}$ at $500{ }^{\circ} \mathrm{C}$ on the surface of the substrate $p$ $\mathrm{Si}$ (001) (a) and Raman spectra (b) $\mathrm{Si} / \mathrm{Ge}$ heterostructure with nanoislands $\mathrm{Si}_{1-\mathrm{x}} \mathrm{Ge}_{\mathrm{x}}$ on the substrate $p$-Si (001) (sample 302.03.11).

The $\mathrm{Si}_{1-\mathrm{x}} \mathrm{Ge}_{\mathrm{x}} / \mathrm{Si}$ heterostructures are refered to the second type, in which the potential well for holes is in the valence band of $\mathrm{Si}_{1-\mathrm{x}} \mathrm{Ge}_{\mathrm{x}}$ (Fig. 2a). The energy diagram of the heterojunction is primarily determined by the values of the band gap and electron affinity of the contacting materials. In unstrained $\mathrm{Si}_{1-\mathrm{x}} \mathrm{Ge}_{\mathrm{x}}$ alloys the bandgap decreases monotonically with increasing of Ge content. Fig. $2 b$ shows the results of numerical calculations of the energy spectra of holes in $\mathrm{Si}_{1-\mathrm{x}} \mathrm{Ge}_{\mathrm{x}}$ quantum wells with the width $2 \mathrm{~nm}$ for a different Ge content. The analysis shows that the energy position of localized states with respect to the top of Si valence band increases nonlinearly with $x$ due to the dependence of the hole effective mass from the strain values in this system. A deep potential well in the valence band favors to accumulation of holes in $\mathrm{Ge}$ nanoislands in the wide temperature range. In other words, the Ge nanoislands can be considered as a giant traps for holes. The positive charge of trapped holes induces downward band bending in the underlying $p-\mathrm{Si}$ substrate. Moreover, the band bending expected to be larger in the region beneath of nanoisland base.

Analyzing the energy diagrams of $\mathrm{Si}_{1-x} \mathrm{Ge}_{\mathrm{x}} / \mathrm{Si}$ heterojunction, we can conclude that the photosensitivity range of these structures is determined by the position of the Fermi level in the heterostructure, i.e. the concentration dopant in Si substrates and epitaxial films (Fig. 2a). Interband optical transitions are realized in the presence of electrons in quantum-sized states of the valence band nanoislands. For intraband transitions in the valence band, the Fermi level must be below at least the ground state of nanoislands. Development of efficient optoelectronic devices requires information on energy, oscillator strengths, and selection rules for interband and intraband transitions. Fluorescence measurements do not reflect all transitions possible in heterogeneous in size and composition of deformations in heterostructures. Opportunities of absorption spectroscopy are severely limited by the fact that the passage of radiation through nanoscale quantum dot layer is absorbed only by its small part $\left(\sim 10^{-4}-10^{-5}\right)$. As a result, the direct measurement of the absorption spectra of quantum dots is rather difficult task, which requires a very sensitive technique and long-time measurements. One of the methods that makes it possible to study the absorption spectra in nanoscale semiconductor structures is an in-plane photocurrent spectroscopy. The value of photoconductivity is proportional to the number of photogenerated charge carriers and, consequently, the absorption coefficient. Photocurrent spectroscopy is a direct, sensitive and relatively simple method of studying the shape of optical absorption spectra and energy and interband transitions possible in heterostructures with nanoscale objects.
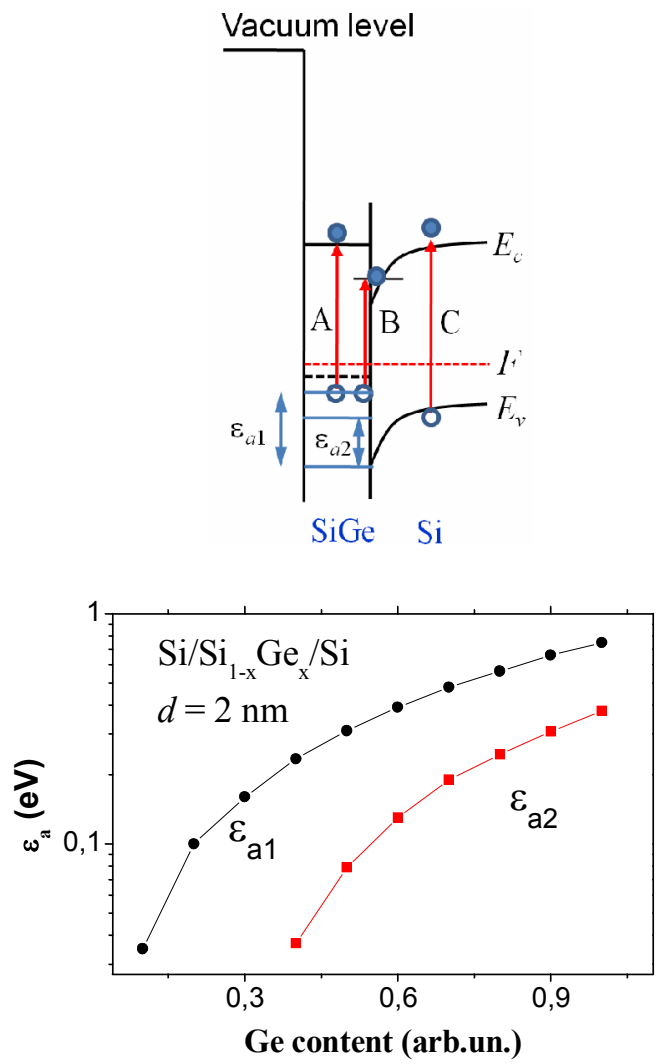

a)

Fig. 2. Energy diagram of $\mathrm{Si} / \mathrm{Ge}$ heterostructures with $\mathrm{Ge}$ nanoislands (a). The activation energies for localized holes of $\mathrm{Si}_{1-\mathrm{x}} \mathrm{Ge}_{\mathrm{x}}$ quantum wells with the width $2 \mathrm{~nm}$ and different content of $\mathrm{Ge}(\mathrm{b})$. 
Excitation of non-equilibrium charge carriers in $\mathrm{Si} / \mathrm{Ge}$ heterostructures with $\mathrm{Ge}$ nanoislands causes conductivity changes in the space charge region of $p$-Si transport channel. Photoconductivity spectra (Fig. 3a) measured at excitation and steady temperatures 50-80$120 \mathrm{~K}$ contained two components. At $h v>\varepsilon_{g, \mathrm{Si}}(1.16 \mathrm{eV}$ at $50 \mathrm{~K}$ ), the main contribution to the photoconductivity gives electron-hole pairs photoexcited in the substrate $p$-Si due to interband transitions (see transition $C$ in Fig. 2a).

In the spectral region where $\mathrm{Si}$ is transparent, photoconductivity originates from interband electronic transitions involving localized states of nanoislands $\mathrm{Si}_{1-}$ ${ }_{x} \mathrm{Ge}_{\mathrm{x}}$. The monopolar photoconductivity was observed in this case. Interband electronic transitions between localized states of the valence band of $\mathrm{SiGe}$ nanoislands and delocalized states of the conduction band of silicon surrounding can be observed in low-dimensional Si-Ge heterostructures. The spectral range of interband transitions is determined by the Ge content of QDs, strain values, and confinement energy for holes in the

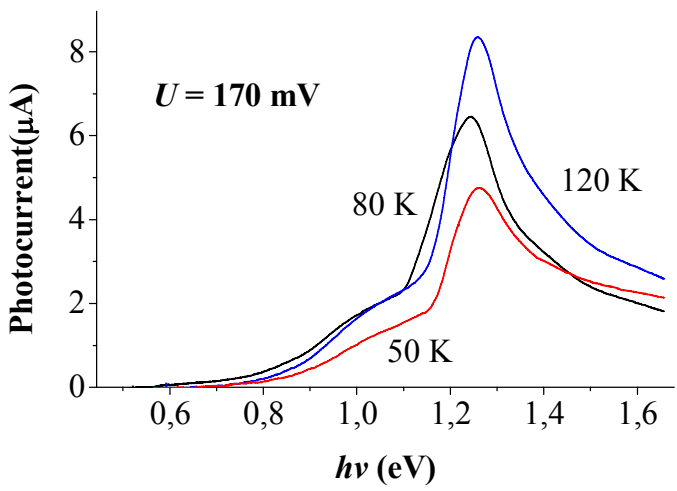

a)

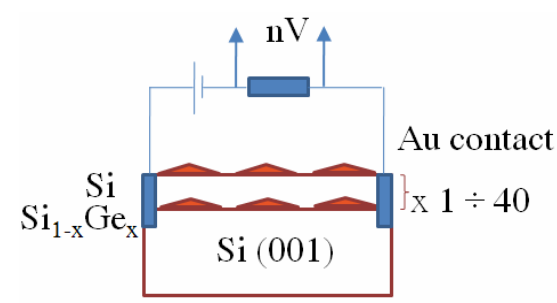

b)

Fig. 3. Photoconductivity spectra of $\mathrm{Si} / \mathrm{Ge}$ heterostructure with nanoislands $\mathrm{Si}_{1-\mathrm{x}} \mathrm{Ge}_{\mathrm{x}}$ on the substrate $p$-Si (001).

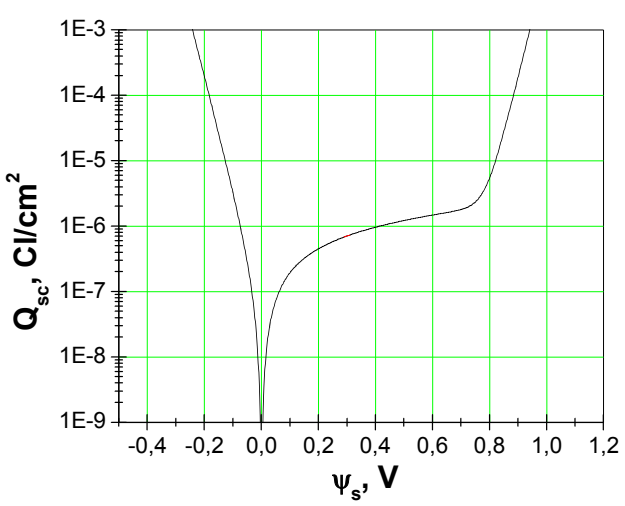

Fig. 4. Dependence of charge in the SCR on the surface potential $\psi_{s}$, calculated for $p$-type silicon. valence band [10]. Transitions A and B (Fig. 2a) are possible, if the ground state is partially filled by electrons. These transitions cause the appearance of nonequilibrium electrons in the Si spacer layers and WLs, which are transport channel, while photoexcited holes are localized in Ge.

The excess of holes in Ge nanoislands induces conductivity changes in the near-surface depletion layer of $p$-Si substrate due to the field-effect. Thus, we have not yet considered the influence of the band bending at the $\mathrm{Si} / \mathrm{Ge}$ and $\mathrm{Si} / \mathrm{SiO}_{2}$ interfaces (i.e., the fixed surface charge density $Q_{s}$ and the bulk doping level) on transport of photoinduced charge carriers. To understand this, here we consider an boron doped $p$-type $\operatorname{Si}(100)$ substrate with $N_{a}$ $=10^{15} \mathrm{~cm}^{-3}$. The surface positive charge produces electric field within the space charge region and a corresponding downward band bending at the silicon surface, following from Poisson equation. Fig. 4 shows dependence of the charge in the space charge region (SCR) $Q_{s c}$ as a function of surface potential $\psi_{s}$ calculated for different concentrations of acceptors for silicon (Si) $p$-type with parameters: $N_{a}=5 \cdot 10^{15} \mathrm{~cm}^{-3}$ and $T=290 \mathrm{~K}$. Surface potential measurements give band bending values about $300 \mathrm{meV}$, i.e. we have deal with a depletion region in the near-surface region of Si substrate.

Change the value of capacity in the SCR is $\frac{d Q_{s c}}{d \Psi_{s}}=2.57 \cdot 10^{-6} \frac{\mathrm{Cl} \cdot \mathrm{cm}^{-2}}{\mathrm{~V}}$. The change in the quantum dot charge per hole will change the surface potential by $0.63 \mathrm{mV}$, which significantly affect the value of surface conductivity due to the field effect.

\section{Conclusions}

In general, the mechanism of photoconductivity in the $\mathrm{Ge} / \mathrm{Si}$ heterosystems, which are referred to the second type heterostructures, depends on the energy of exciting illumination quantum. The lateral photoconductivity observed within the range $0.63-1.0 \mathrm{eV}$ below the fundamental absorption edge of $c$-Si was caused by interband transitions from the ground state of $\mathrm{Ge}$ nanoislands to the conduction band of silicon surrounding. Photoexcited holes were found to be localized in Ge nanoislands, while photoelectrons are supposed to be free in the conduction band of Si giving contribution to the monopolar photoconductivity. The positive charge of trapped holes induces conductivity changes in the near-surface depletion layer of $p$-Si substrate due to the field-effect.

\section{References}

1. K. Brunner, Si/Ge nanostructures // Repts. Progr. Phys. 65, p. 27-72 (2002).

2. O.G. Schmidt and K. Eberl // Phys. Rev. B, 61, p. 13721 (2000). 
3. D. Bimberg, M. Grundmann, N. Ledentsov, Quantum Dot Heterostructures. John Wiley \& Sons, Ltd., Chichester, 1999.

4. C. Miesner, O. Röthig, K. Brunner, G. Abstreiter, Intra-valence band photocurrent spectroscopy of self-assembled Ge dots in Si // Appl. Phys. Lett. 76, p. 1027-1029 (2000).

5. H. Lafontaine, N.L. Rowell, S. Janz, D.-X. Xu, Growth of undulating $\mathrm{Si}_{0.5} \mathrm{Ge}_{0.5}$ layers for photodetectors at $\lambda=1.55 \mu \mathrm{m} / / \mathrm{J}$. Appl. Phys. 86, p. 1287-1291 (1999).

6. Y. Chen, Y.F. Lu, L.J. Tang, Y.H. Wu, B.J. Cho, X.J. Xu, J.R. Dong, W.D. Song, Annealing and oxidation of silicon oxide films prepared by plasma-enhanced chemical vapor deposition // J. Appl. Phys. 97, p. 014913 (2005) .
7. P. Sutter, E. Mateeva-Sutter, L. Vescan, Oblique stacking of three-dimensional dome islands in Ge/Si multilayers // Appl. Phys. Lett. 78(12), p. $1736-1738$ (2001).

8. S.V. Kondratenko, Yu.N. Kozyrev, M.Yu. Rubezhanska, V.S. Lysenko, Morphology and photoelectric properties of Si-Ge quantumsized structures et al. // Intern. Workshop "In situ characterization of near-surface processes", Eisenerz, 2010, May 30 -June 3: Abstract of Book, Eisenerz, Austria, 2010, p. 40.

9. S.V. Kondratenko, A.S. Nikolenko, O.V. Vakulenko, M.Ya. Valakh, V.O. Yukhymchuk, A.V. Dvurechenskii, A.I. Nikiforov // Nanotechnology, 19, 145703 (2008).

10. S.V. Kondratenko, S.L. Golovinskiy, A.S. Nikolenko and O.V. Vakulenko // Semiconductor Science and Technology, 21(7), p. 857 (2006). 